\title{
EQUILÍBRIO ASSOCIADO AO RISCO DE QUEDAS EM IDOSOS NÃO INSTITUCIONALIZADOS
}

\author{
EQUILIBRIUM ASSOCIATED WITH THE RISK OF FALLS IN NON- \\ INSTITUTIONALIZED ELDERLY
}

\author{
Andressa Porto Dutra, Kleyton Trindade Santos
}

Faculdade Independente do Nordeste - FAINOR

\begin{abstract}
Objective: to carry out an integrative review of the literature on the relationship between balance and risk of falls in the elderly population. Methods: this is an integrative review of the literature between 2007 and 2016, which occurred through consultation with the Virtual Health Library (VHL) using the descriptors "accident by falls", "elderly" and "postural equilibrium" , Which were separated by the Boolean operator "AND", with 7 studies being selected. Results: in relation to the balance in the elderly associated to the risk of falls and to the increase of this occurrence, the 7 articles maintained a relation in the approach when they showed that the changes in the balance generate the Predisposition and increases the risk of falls in the elderly population. Conclusions: it is necessary to identify early risks for the prevention of falls, and in this way, to take care of preventive actions that stimulate the responsibility of selfcare, Better quality of life and the exercise of autonomy among these individuals.
\end{abstract}

Keywords: Accidents by falls; Old man; Postural balance.

\section{Resumo}

Objetivo: realizar uma revisão integrativa da literatura sobre a relação existente entre $o$ equilíbrio e o risco de queda da população idosa. Métodos: trata-se de uma revisão integrativa da literatura entre os anos de 2007 a 2016, que ocorreu por meio de consulta à Biblioteca Virtual em Saúde (BVS) utilizando os descritores "acidentes por quedas", "idoso" e "equilibrio postural", separados pelo operador boleano "AND", sendo selecionados 7 estudos. Resultados: em relação ao equilíbrio em idosos associado ao risco de quedas e ao aumento dessa ocorrência, os 7 artigos mantiveram uma relação na abordagem quando evidenciaram que as alterações no equilíbrio geram a predisposição $e$ aumenta o risco de quedas na população idosa. Conclusões: percebe-se que é necessária a identificação precoce dos riscos para a prevenção de quedas, e desta forma, atentar-se para a realização de ações preventivas que estimulem a responsabilidade do autocuidado, melhor qualidade de vida e o exercício da autonomia entre esses indivíduos.

Palavras-chave: Acidentes por quedas; Idoso; Equilíbrio

postural. 


\section{Introdução}

O aumento da expectativa de vida e o envelhecimento merecem atenção, estando relacionados à rápida diminuição das taxas de natalidade observada nos últimos anos o que aponta para um crescente número da população de idosos, principalmente em países em desenvolvimento, como é o caso do Brasil, o que faz com que, segundo estimativas, em 2025 o país chegue a uma população de 32 milhões de idosos ${ }^{1}$, que em alguns casos terão como característica a ocorrência de limitações que geralmente afetam o desenvolvimento de suas atividades ${ }^{2}$.

O envelhecimento é um processo complexo que abrange questões psicológicas e sociais do desenvolvimento humano, sendo capaz de gerar incapacidades funcionais que podem desencadear eventos como as quedas, definidas através de um conjunto de ações de forças físicas que agem em um corpo, resultando em uma instabilidade do centro de equilíbrio que pode levar o indivíduo a cair da própria altura ou de um nível mais alto ${ }^{3}$.

O sistema nervoso é uma das principais áreas acometidas pelo processo de envelhecimento levando a mortes e perdas de neurônios que se localizam em áreas nobres responsáveis pelo planejamento do movimento, equilíbrio corporal e a coordenação motora ${ }^{4}$.

Neste contexto, os distúrbios do equilíbrio corporal geram manifestações de grande impacto para os idosos, gerando a predisposição a quedas, fraturas, sofrimento, imobilidade corporal, medo de um novo evento de queda e altos custos com o tratamento de saúde, reduzindo a suas atividades de vida diária impactando de maneira negativa a qualidade de vida da população idosa ${ }^{4}$.

As quedas são um problema iminente a saúde dos idosos, sendo destacados como um dos principais problemas clínicos e de saúde pública dos países em desenvolvimento por apresentarem uma elevada incidência, comprometendo a saúde psíquica e física do idoso, interferindo diretamente na sua condição de saúde e envelhecer ${ }^{5}$. Estudos mostram que $50 \%$ das quedas causadas em pessoas idosas resultam em algum tipo de injuria, sendo que $36 \%$ a $51 \%$ dessas são injurias graves ${ }^{6}$.

Diante do exposto, e considerando o aumento da ocorrência de quedas na saúde do idoso bem como as suas consequências, se faz importante identificar os fatores de risco para as quedas e sua relação com o desequilíbrio corporal para desenvolver possíveis intervenções eficazes e planejamento de ações preventivas. Portanto, o presente estudo teve como objetivo realizar uma revisão integrativa da literatura no período de 2007 a 2016, por meio da seleção e análise de artigos científicos que abordaram as relações existentes entre o equilíbrio e o risco de queda da população idosa.

\section{Metodologia}

Trata-se de uma revisão integrativa que busca investigar a relação entre quedas e equilíbrio em idosos. Este método tem por finalidade reunir e sintetizar resultados de pesquisas sobre um delimitado tema, de forma sistemática e ordenada, contribuindo para o entendimento aprofundado acerca do mesmo ${ }^{7}$.

Para o desenvolvimento do estudo, foram seguidas as seis etapas características para construção de uma revisão integrativa: 1) identificação do tema ou questão de pesquisa; 2) estabelecimento de critérios para inclusão e exclusão de estudos; 3) definição das informações a serem extraídas dos estudos selecionados; 4) avaliação dos estudos incluídos; 5) interpretação dos resultados; e 6) apresentação da revisão ${ }^{7}$.

A investigação exploratória dos estudos ocorreu entre outubro e novembro de 2016, por meio de consulta à Biblioteca Virtual em Saúde (BVS). Os termos utilizados para a busca, selecionados a partir dos Descritores em Ciências da Saúde (DeCS), da Literatura Latino-Americana e do Caribe em Ciências da Saúde (LILACS), foram "acidentes por quedas", "idoso" e "equilíbrio postural", todos eles separados pelo operador boleano AND.

No presente estudo adotou-se como critério de inclusão: documento do tipo artigo científico original, com disponibilidade de texto completo, publicado nos últimos 10 anos (2007 a 2016) que abordasse o tema queda e equilíbrio como eixo central.

A busca inicial seguindo esses passos identificou um total de 39 artigos, sendo que, após a leitura criteriosa dos títulos pelos dois autores do presente manuscrito, 15 foram descartados, restando 24. Estes, por sua vez, tiveram seus resumos lidos na íntegra $\mathrm{e}$ passaram por uma leitura flutuante de todo o corpo do estudo, a fim de identificar quais abordavam, de forma central, a temática estudada e que, desta forma, atendiam aos 
critérios de inclusão do presente estudo. Ao final desta etapa, restaram 7 artigos, julgados coerentes com a proposta desta revisão.

Tais estudos representaram os artigos que foram sintetizados na presente revisão, sendo que foram lidos e fichados em tabela com a síntese das principais informações, tais como autores, ano de publicação, objetivos e principais resultados.

A Figura 1 apresenta os principais elementos do delineamento metodológico desta revisão integrativa, no que se refere à busca e à análise crítica dos artigos.

Figura 1. Representação esquemática do delineamento metodológico do estudo, Vitória da Conquista, BA, 2017.

Busca eletrônica na Biblioteca Virtual em saúde (MEDLINE, LILACS $\sqrt{2}$

Descritores: "Acidentes por quedas"; "idoso"; "equilibrio postural"

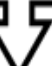

Identificação de 39 artigos científicos

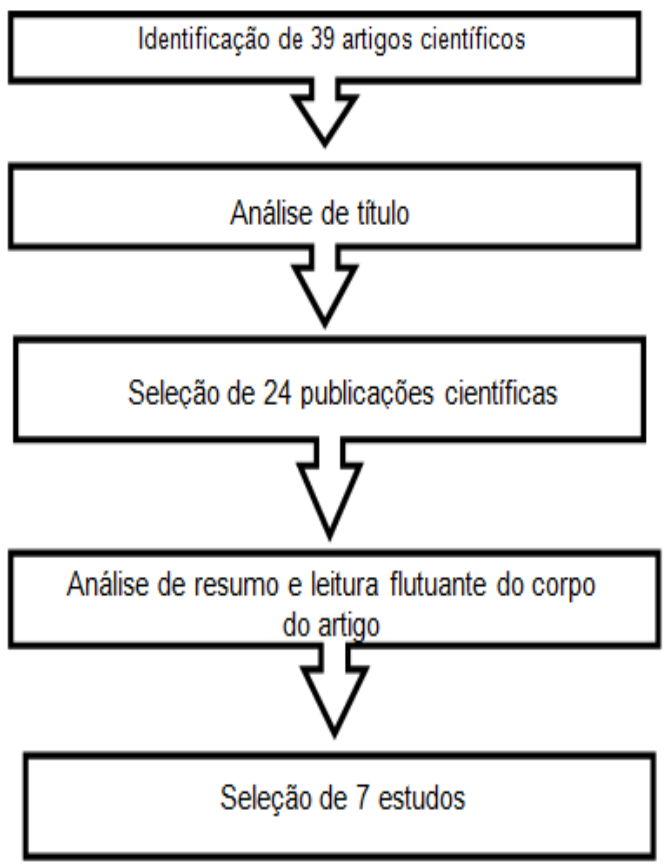

Fonte: Elaboração própria

\section{Resultados e Discussões}

No presente estudo, o risco de queda foi abordado pelos 7 artigos analisados onde ressaltaram a importância da identificação precoce desse risco para a prevenção de quedas e uma melhor qualidade de vida desses idosos.

No que se refere a prevalência de quedas apenas 1 (14,28\%) explorou esse indicador em sua população estudada sendo que 32 idosos $(25,2 \%)$ relataram ter caído pelo menos uma vez no ano tendo como prevalência de quedas $25,20 \%$.

Em relação aos fatores associados ao risco de quedas $4(57,14 \%)$ destacaram o sexo feminino, três $(42,9 \%)$ alterações da marcha, dois $(28,57 \%)$ o sedentarismo, três $(42,9 \%)$ a não prática de atividade física e três (42,9\%) evento de quedas pregressas.

No que diz respeito à questão do equilíbrio em idosos associado ao risco de quedas, os 7 artigos mantiveram uma relação na abordagem quando evidenciaram que as alterações no equilíbrio geram a predisposição e aumenta o risco de quedas na população idosa. Cinco $(71,42 \%)$ dos artigos trabalharam de maneira isolada a identificação do déficit de equilíbrio associado com o risco de queda pela aplicação da escala de equilíbrio de Berg, Timed Up and Go Test, sentado para de pé, step e equilíbrio estático, enquanto que dois $(28,57 \%)$ avaliarão fatores associados ao risco de queda onde evidenciaram através do teste de alcance funcional, Escala de Tinetti e o Dynamic Gait Index que o fator mais significante e preditor deste evento é o déficit de equilíbrio.

Observando a utilização dos testes e escalas para identificação de fatores de risco de quedas, percebeu-se que quatro $(57,14 \%)$ aplicaram a escala de equilíbrio de Berg, sendo no presente estudo a mais utilizada na avaliação do equilíbrio, em segundo dois $(28,57 \%)$ utilizaram o Timed Up and Go Test que avalia o equilíbrio dinâmico, um $(14,29 \%)$ o Dynamic Gait Index, um $(14,29 \%)$ o Teste do alcance funcional, um (14,29\%) a Escala de Equilíbrio e Marcha de Tinetti, um (14,29\%) step teste, velocidade de marcha de seis metros e equilíbrio estático na posição semi-tandem.

As demais informações e a síntese dos resultados e conclusões dos artigos encontramse presentes no Quadro 1.

O crescimento populacional de idosos é uma realidade vivenciada em todo mundo, e no Brasil, o Ministério da Saúde define como umas das prioridades a atenção ao idoso.

Ao proceder com as análises individuais de cada artigo encontrado, observou-se que apenas um deles fazia menção sobre dados de prevalência de quedas sucedidas em um ano na população idosa estudada, mostrando que 25,2 $\%$ dos idosos sofreram ao menos uma queda no último ano. Esses resultados são semelhantes ao estudo de Siqueira et al $^{8}$ em que avaliou 4003 idosos e encontrou uma prevalência de $34,8 \%$. Conhecer a prevalência de quedas é de extrema 
Quadro 1 - Referência, objetivo, principais resultados e conclusões dos artigos selecionados do estudo. Vitória da Conquista-BA, Brasil, 2017.

\begin{tabular}{|c|c|c|c|}
\hline $\mathbf{N}^{\circ}$ & Referências & Objetivos & $\begin{array}{l}\text { Principais resultados e } \\
\text { conclusões }\end{array}$ \\
\hline 1. & $\begin{array}{l}\text { CASTRO, P. M. M. A. et } \\
\text { al. Testes de equilíbrio } \\
\text { e mobilidade funcional } \\
\text { na predição e } \\
\text { prevenção } \\
\text { de riscos de quedas em } \\
\text { idosos. Rev. Bras. } \\
\text { Geriatr. Gerontol., Rio } \\
\text { de Janeiro, 2015; v. 18, } \\
\text { n.1. }\end{array}$ & $\begin{array}{l}\text { Avaliar a ocorrência de } \\
\text { quedas e seus } \\
\text { fatores associados e } \\
\text { identificar os pontos de } \\
\text { corte de testes de } \\
\text { equilíbrio e mobilidade } \\
\text { funcional mais adequados } \\
\text { para identificar idosos } \\
\text { ativos na comunidade com } \\
\text { risco de quedas. }\end{array}$ & $\begin{array}{l}\text { - A ocorrência de quedas apresentou associação } \\
\text { apenas com a variável "gênero", sendo maior a } \\
\text { chance de cair entre as mulheres. } \\
\text { - Os pontos de corte para os testes sentado para } \\
\text { de pé, step teste e velocidade da marcha foram } \\
8,5 \text { segundos, } 7,5 \text { segundos e } 0,98 \mathrm{~m} / \mathrm{s} \text {, } \\
\text { respectivamente. } \\
\text { - Os resultados demonstraram ser importante } \\
\text { adequar os pontos de corte dos testes de } \\
\text { equilíbrio e mobilidade ao perfil da população } \\
\text { avaliada. } \\
\text { - Sem essa adequação, os testes têm sua } \\
\text { sensibilidade e especificidade comprometidas na } \\
\text { identificação de idosos com risco de cair. }\end{array}$ \\
\hline 2. & $\begin{array}{l}\text { ALVES, L. V. et al. } \\
\text { Avaliação da tendência } \\
\text { à quedas em idosos de } \\
\text { Sergipe. Rev. CEFAC. } \\
2014 \text { Set-Out; v. } 16, \mathrm{n} \text {. } \\
5 .\end{array}$ & $\begin{array}{l}\text { Analisar o desempenho de } \\
\text { idosos no teste Dynamic } \\
\text { Gait Index e Timed Up and } \\
\text { Go Test para avaliar a } \\
\text { tendência para quedas e } \\
\text { verificar a interferência } \\
\text { das variáveis idade, } \\
\text { gênero e relato de eventos } \\
\text { de quedas pregressas. }\end{array}$ & $\begin{array}{l}\text { - Verificou-se que } 37(61,7 \%) \text { dos } 60 \text { voluntários } \\
\text { pontuaram com índices inferiores ao padrão de } \\
\text { normalidade no Timed Up and Go Test, enquanto, } \\
\text { no Dynamic Gait Index } 19 \text { ( } 31,7 \%) \text { voluntários } \\
\text { apresentaram índices inferiores ao ponto de } \\
\text { corte. } \\
\text { - Ocorreu associação estatisticamente significante } \\
\text { entre Dynamic Gait Index e as variáveis idade e } \\
\text { quedas. Neste estudo o Timed Up and Go Test } \\
\text { apresentou associação com as variáveis quedas e } \\
\text { gênero. } \\
\text { - Foi frequente a constatação de alteração } \\
\text { funcional de equilíbrio e de marcha, e a maioria } \\
\text { da amostra apresentou tendência para quedas. } \\
\text { - A idade associou-se com alterações de marcha e } \\
\text { equilíbrio, o gênero com tendência para quedas, } \\
\text { e os relatos de eventos de quedas pregressas } \\
\text { com os dois instrumentos aplicados. }\end{array}$ \\
\hline 3. & $\begin{array}{l}\text { MÜJDECI, B. et } \\
\text { al.Avaliação do } \\
\text { equilíbrio em idosos } \\
\text { que sofrem queda e } \\
\text { aqueles que não } \\
\text { sofrem quedas. } \\
\text { Brazilian Journal of } \\
\text { Otorhinolaryngology } \\
\text { v. 78, n.5, } \\
\text { setembro/Outubro } \\
2012\end{array}$ & $\begin{array}{l}\text { Avaliar o equilíbrio } \\
\text { entre idosos que caem } \\
\text { e aqueles que não } \\
\text { sofrem queda. Desenho } \\
\text { do estudo: clínico. }\end{array}$ & $\begin{array}{l}\text { - Os valores de Teste de Organização } \\
\text { Sensorial (TOS) 3, } 6 \text { e composto, Escala de } \\
\text { Equilíbrio de Berg (EEB) e escore de } \\
\text { velocidade no eixo direita-esquerda do } \\
\text { Deslocamento Ponderal Rítmico (DPR) foram } \\
\text { significativamente menores no Grupo I (que } \\
\text { sofreram queda em um período de um ano) } \\
\text { e determinamos o TOS } 4,5,6 \text {, composto e } \\
\text { EEB do grupo II (não tinham passado de } \\
\text { quedas) ( } p<0,05 \text { ). } \\
\text { - Os valores da Posturografia Dinâmica } \\
\text { Computadorizada (PDC) e EEB em idosos } \\
\text { que sofrem quedas estiveram } \\
\text { significativamente mais baixos quando } \\
\text { comparados àqueles que não caíram. }\end{array}$ \\
\hline
\end{tabular}




\begin{tabular}{|c|c|c|c|}
\hline 4. & $\begin{array}{l}\text { SANTOS, G. M. et al. } \\
\text { Valores preditivos para } \\
\text { o risco de queda em } \\
\text { idosos praticantes e não } \\
\text { praticantes de atividade } \\
\text { física por meio do uso } \\
\text { da Escala de Equilíbrio } \\
\text { de Berg. Ver Bras } \\
\text { Fisioter.2011; v. 15, n. } 2 \text {. }\end{array}$ & $\begin{array}{l}\text { Analisar os } \\
\text { preditivos para o risco de } \\
\text { queda em idosos } \\
\text { praticantes e não- } \\
\text { praticantes de atividade } \\
\text { física por meio do uso da } \\
\text { EEB. }\end{array}$ & $\begin{array}{l}\text { - Evidenciou-se que a média do escore da EEB nos } \\
\text { praticantes de atividade física foi de } 54,7 \text { pontos; } \\
\text { enquanto que, entre os não-praticantes, foi de } \\
50,8 \text {, obtendo-se diferença significativa entre os } \\
\text { grupos ( } \rho=0,001) \text {. } \\
\text { - Nos sujeitos não-praticantes de atividade física, } \\
\text { o melhor ponto de corte foi em } 49 \text { pontos, } \\
\text { apresentando } S \text { de } 91 \% \text { e E de } 92 \% \text {, enquanto a } \\
\text { EEB apresentou baixa S, variando entre } 0 \% \text { e } 15 \% \text {. } \\
\text { Variando entre } 83 \% \text { e } 100 \% \text { nos sujeitos } \\
\text { praticantes de atividade física regular nos pontos } \\
\text { de corte analisados. } \\
\text { - A escala não alcançou S suficiente para } \\
\text { diferenças individuais entre idosos com altos } \\
\text { níveis de capacidade de equilíbrio dentre aqueles } \\
\text { que praticam atividades físicas regularmente. }\end{array}$ \\
\hline 5. & $\begin{array}{l}\text { GAl, J. et al. } \text { Fatores } \\
\text { associados a quedas em } \\
\text { mulheres } \quad \text { idosas } \\
\text { residentes na } \\
\text { comunidader Rev Assoc } \\
\text { Med Bras 2010; v. 56, n. } \\
3 .\end{array}$ & $\begin{array}{l}\text { Verificar quais os fatores } \\
\text { associados à presença de } \\
\text { queda em um grupo de } \\
\text { mulheres idosas } \\
\text { independentes } \\
\text { autônomas. }\end{array}$ & $\begin{array}{l}\text { - Não se encontrou relação estatisticamente } \\
\text { significativa entre idade, fatores } \\
\text { sociodemográficos, tontura, medicação } \\
\text { psicotrópica, má autopercepção da saúde e da } \\
\text { visão e presença de depressão com o fenômeno } \\
\text { queda. Entretanto, houve relação de significância } \\
\text { estatística para o Teste do Alcance Funcional e a } \\
\text { Escala de Equilíbrio e Marcha de Tinetti. } \\
\text { - A condição de equilíbrio corporal apresentada } \\
\text { pelas mulheres idosas foi o fator que teve } \\
\text { associação coma ocorrência de quedas. } \\
\text { - O Teste do Alcance Funcional e a Escala de } \\
\text { Tinetti foram consideradas eficientes na predição } \\
\text { do risco de cair, o que sugere que podem ser } \\
\text { utilizados tanto para avaliação quanto para } \\
\text { identificação de melhora do equilíbrio corporal } \\
\text { após o treinamento. }\end{array}$ \\
\hline 6. & $\begin{array}{l}\text { PIMENTEL, R. M. } \\
\text { SCHEICHER, M. E. } \\
\text { Comparação do risco de } \\
\text { queda em idosos } \\
\text { sedentários e ativos por } \\
\text { meio da escala de } \\
\text { equilíbrio de Berg. } \\
\text { Fisioter Pesq. 2009; v. } \\
\text { 16, n. 1. }\end{array}$ & $\begin{array}{l}\text { Este estudo visou } \\
\text { comparar o risco de } \\
\text { quedas entre idosos } \\
\text { sedentários e ativos, } \\
\text { verificando como a prática } \\
\text { de exercício físico se } \\
\text { reflete no desempenho } \\
\text { dos sujeitos na escala de } \\
\text { Berg. }\end{array}$ & $\begin{array}{l}\text { - Os escores médios na escala de Berg dos grupos } \\
\text { sedentário e ativo foram } 47,7 \pm 5,6 \text { pontos e } \\
53,6 \pm 3,7 \text {, respectivamente }(p<0,0001) \text {. } \\
\text { - A análise dos escores evidenciou que o grupo } \\
\text { sedentário apresentou } 15,6 \text { vezes mais risco de } \\
\text { quedas do que o grupo ativo ( } p=0,002) \text {. } \\
\text { - O desempenho na escala de Berg foi pior no } \\
\text { grupo sedentário do que no ativo, sugerindo que } \\
\text { a prática regular de atividades físicas pode } \\
\text { interferir nesse desempenho e que os sujeitos } \\
\text { ativos têm menor risco de queda. }\end{array}$ \\
\hline 7. & $\begin{array}{l}\text { RESENDE, S. M. et al. } \\
\text { Efeitos da hidroterapia } \\
\text { na recuperação do } \\
\text { equilíbrio e prevenção } \\
\text { de quedas em idosas. } \\
\text { Rev Bras Fisioter. 2008; } \\
\text { v. } 12 \text {, n. } 1 .\end{array}$ & $\begin{array}{l}\text { Avaliar o efeito de um } \\
\text { programa de hidroterapia } \\
\text { no equilíbrio e no risco de } \\
\text { quedas em idosas. }\end{array}$ & $\begin{array}{l}\text { - A hidroterapia promoveu aumento significativo } \\
\text { do equilíbrio das idosas, avaliado por meio da } \\
\text { Escala de Equilíbrio de Berg }(p<0,001) \text { e teste } \\
\text { Timed Up \& Go }(p<0,001) \text {, e ainda, redução do } \\
\text { risco de quedas }(p<0,001) \text {, de acordo com o } \\
\text { modelo de Shumway-Cook et al. } \\
\text { - Pode-se sugerir que este programa de } \\
\text { hidroterapia para equilíbrio aumentou o } \\
\text { equilíbrio e reduziu o risco de quedas nas idosas. }\end{array}$ \\
\hline
\end{tabular}

Fonte: Elaboração própria

No que se refere aos fatores associados ao risco de queda, identificamos que em quatro artigos foi possível observar que o sexo feminino esteve associado com as quedas, corroborando com o estudo de Antes et al $^{9}$ que contou com a participação de 1.705 idosos e identificou uma maior prevalência de quedas no sexo feminino. Santos et al $^{10}$ reforça esse dado, ao identificar em seu estudo a ocorrência maior de quedas no sexo feminino justificado pela fragilidade, maior prevalência de doenças crônicas e exposição às atividades domésticas que podem impor risco. 
Outro fator citado como associado ao risco de quedas foi à alteração da marcha mostrada em três artigos (42,9\%). Destes, Alves et al ${ }^{11}$ observou que de 60 voluntários, 37 (61,7\%) enquadravam-se no grupo com alto risco para quedas no TUGT por apresentarem uma marcha lenta, cautelosa, arrastada, acompanhada do gingado, ocasionando maior propensão às quedas. Para Gai et $a^{12}$, após avaliar em 83 idosas obteve na aplicação na Escala de Equilíbrio e Marcha de Tinetti a pontuação menor que 19 em $16 \%$ das idosas tendo relação com maior chance de cair.

A partir desses achados, nota-se que a alteração da marcha se enquadra como fator de risco para os idosos, onde o envelhecimento está associado a modificações no seu padrão gerando instabilidade e déficit na habilidade motora, sendo que algumas dessas alterações em idosos não são mudanças adaptativas, e sim limitações específicas relacionadas a idade.

Coqueiro et $\mathrm{al}^{13}$ demonstram que $55 \%$ das quedas em idosos estão relacionados com distúrbios da marcha, que podem ser desde a diminuição da velocidade até a falta de sinergismo muscular assemelhando ao resultado destacado por Gervásio et $a^{14}$ onde os idosos apresentam uma marcha mais lenta por redução da velocidade como consequência primária do envelhecimento, onde a mesma é responsável por $48 \%$ das quedas em idosos decorrente de tropeço durante a fase de balanço e a não elevação adequada do pé.

Dois artigos desta revisão destacaram o sedentarismo como fator de risco a quedas, onde Santos et al $^{15}$ observou que dentre os praticantes de atividade física regular, 23 (25,2\%) apresentaram auto-relato de queda no último ano, enquanto, no grupo não-praticante, a incidência foi de 37 (38,5\%). No estudo de Pimentel et $a^{16}$ avaliaram 70 idosos onde os escores médios na escala de Berg do grupo sedentário foi 47,7 , enquanto que no grupo ativo foi 53,6 .

Nesse mesmo estudo a análise dos escores evidenciou que o grupo sedentário apresentou 15,6 vezes mais risco de quedas do que o grupo ativo, afirmando que a população idosa sedentária possui mais probabilidade de um possível evento de queda, sendo uma preocupação relevante, pois os idosos sedentários possuem uma diminuição da capacidade funcional e esse déficit tem papel significativo no aumento da incidência de quedas diferente dos idosos praticantes de atividade física. Condizendo com o estudo de Siqueira ${ }^{8}$ onde os seus resultados demonstraram que 0 sedentarismo se associou positivamente com a prevalência e ocorrência de quedas.

Verificou-se que a ocorrência de quedas pregressas aumenta a chance de o idoso cair novamente, sendo esses resultados destacados em três artigos desta revisão. Müjdeci, Aksoy e Atas $^{17}$ buscaram avaliar 30 idosos através das médias dos valores na Escala de Equilíbrio de Berg, dividindo esses idosos em dois grupos: aqueles que sofrem quedas em um ano, e aqueles sem quedas pregressas. Os autores observaram que aqueles idosos sem quedas pregressas tiveram 6,7 pontos a mais na média do que idosos caidores (54,6 pontos e 47,9 pontos, respectivamente), sendo esse achado estatisticamente significativo $(p<0,05)$.

O outro estudo encontrado na revisão que analisou o histórico de quedas com o medo de cair, foi o de Gai et $\mathrm{al}^{12}$, onde identificou que das 83 idosas participantes $51,8 \%(n=43)$ relataram ter sofrido queda no último ano. A ocorrência dessas quedas anteriores incapacita a mobilidade do idoso e sua integridade emocional como no estudo de Falsarela et $\mathrm{al}^{18}$, que aponta que o medo de cair afeta $73 \%$ dos idosos que sofreram eventos de quedas no anterior resultando em um ciclo vicioso, levando a redução da capacidade funcional, perda da confiança em si mesmo, restrição das atividades, pior qualidade de vida e maior suscetibilidade do idoso a sofrer novas quedas.

Todos os estudos selecionados da nossa revisão indicaram como informação preocupante a presença do comprometimento do equilíbrio estático e dinâmico na população idosa, sendo que 4 (57,14\%) artigos abordaram e avaliaram alterações do equilíbrio estático e dinâmico, 2 $(28,5 \%)$ somente o equilíbrio dinâmico e 1 $(14,28 \%)$ o equilíbrio estático. Todos se apresentaram abaixo dos parâmetros de normalidade, mostrando que o envelhecimento traz a perda de equilíbrio sendo essa alteração fonte de morbidade e mortalidade em pessoas com idade superior a 75 anos.

Esse achado acima citado torna-se ainda mais preocupante quando entende-se que o déficit de equilíbrio predispõe o idoso a um maior risco de queda. Coqueiro et al $^{13}$ afirma em seu estudo que comprometimento do equilíbrio esta associado como maior fator de queda em idosos, afetando de maneira negativa a sua saúde e independência funcional. Essa afirmação é reforçada pelo estudo de Gomes et al $^{19}$ ao identificar que as alterações de equilíbrio estático e dinâmico geram a instabilidade dos 
movimentos impactando de maneira negativa na funcionalidade do idoso aumentando o risco de queda.

Ainda no presente estudo podemos observar a utilização de escalas e testes em sete artigos para avaliar o equilíbrio, velocidade e agilidade do idoso sendo um fator para prevenção do risco de queda. Destacado em quatro artigos como sendo o mais utilizado a escala de equilíbrio de Berg é um método avaliativo de grande eficácia como ressalta o estudo de Ferrarezi ${ }^{20}$ onde aplicaram a escala de equilíbrio de Berg em 172 idosos buscando identificar fatores de risco, pois se sabe que a queda traz limitações para o cotidiano do idoso como a dificuldade na execução dos serviços domésticos, prejuízo no caminhar e a necessidade de ajuda para realizar a higiene pessoal. Esse dado demonstra que quanto menor o equilíbrio, maior é a dificuldade na realização das Atividades de Vida Diária (AVD's) para o idoso.

Dois artigos desta revisão utilizaram o Timed Up and Go Test, sendo um método de avaliação do equilíbrio dinâmico que indica um possível risco de queda, que se detectado precocemente poderá contribuindo para sua prevenção. Bretan et $\mathrm{al}^{21}$ ao analisar a aplicação dos testes, verificou que a escala de equilíbrio de Berg e de mobilidade de Tinetti são amplamente utilizados, mas, exigem maior treinamento de quem aplica e necessitam de espaço e arranjos, enquanto que o Timed Up and Go Test é de fácil aplicação avaliando a mobilidade e o equilíbrio dos indivíduos ao criar um risco de queda propiciado pelo levantar, caminhar e girar o corpo, sentarse, esses são os movimentos solicitados para a realização.

Na presente revisão também verificamos a existência de outros testes para avaliação do risco de quedas tais como Dynamic Gait Index, teste de alcance funcional, step teste, velocidade de marcha de seis metros e equilíbrio estático na posição semi-tandem, porém, são pouco utilizados por outros autores.

A partir dos resultados encontrados nos 7 artigos que compõe esta revisão, foi possível verificar a necessidade da identificação dos episódios de queda e deficiência no equilíbrio, principalmente a partir de testes que podem ser utilizados em diversos ambientes, que vão desde o domicílio até a Unidade Básica de Saúde ${ }^{22}$, fazendo com que a partir desse conhecimento possam ser desenvolvidas ações de promoção e de prevenção da saúde para a melhoria da qualidade de atenção prestada a essa população.

\section{Conclusões}

Com base nos estudos revisados, pode-se concluir que é o déficit de equilíbrio em idosos é algo bastante prevalente e que está associado de maneira direta com o aparecimento de quedas nesses indivíduos.

Percebe-se que mais estudos devem ser realizados buscando a identificação e prevenção dos riscos de quedas principalmente, aqueles relacionados a comprometimento do equilíbrio.

Desta forma Entende-se que é de extrema importância a realização do incentivo de ações que auxiliem no desenvolvimento de ações educativas associadas à prevenção e à realização de exercícios que possibilitem uma marcha segura e aumentem o equilíbrio estático e dinâmico dessa população, a fim de proporcionar e manter a estabilidade de movimento e a funcionalidade do idoso.

\section{Referências}

1. Nicolussi, AC et al. Qualidade de vida em idosos que sofreram quedas: revisão integrativa da literatura. Ciência \& Saúde Coletiva, 2012; 17(3): 723-30.

2. Becker AC, Falcão DVS. O envelhecimento, a velhice e o significado de ser avô (ó) na perspectiva de atores profissionais idosos. Rev. Bras. Geriatr. Gerontol, Rio de Janeiro, 2016; 19(2): 289-302.

3. Almeida VC, Meira SS, Gomes FV, Souza MV, Santos VC, Anjos KF. Qualidade de vida em idosos que sofreram quedas. Rev. APS. 2014 out/dez; 17(4): 530 - 536.

4. Santos IR, Carvalho RC, Lima KBSP, Silva SC, Ferreira AS, Vasconcelos NN. et al. Análise dos parâmetros da marcha e do equilíbrio dos idosos após exercícios aeróbicos e terapêuticos. Arq. Ciênc. Saúde UNIPAR, Umuarama, 2016 jan./abr; 20(1):19-23.

5. Júnior NFP, Santo SMA. Epidemiologia do evento queda em idoso: traçado histórico entre os anos de 2003 e 2012. Rev Min Enferm. 2015 out/dez; 19(4): 994-1004.

6. Nogueira, A et al. Risco de queda nos idosos: educação em saúde para melhoria da qualidade de vida. Revista Práxis, 2013; 4(8).

7. Mendes KDS, Silveira RCCP, Galvão CM. Revisão integrativa: método de pesquisa para a incorporação de evidências na saúde e na enfermagem. Texto Contexto Enferm, Florianópolis, 2008 Out-Dez; 17(4): 758-64. 
8. Siqueira FV, Facchini LA, Piccini RX, Tomasi E, Thumé E, Silveira DS. et al. Prevalência de quedas em idosos e fatores associados. Rev Saúde Pública 2007;41(5):749-56.

9. Antes DL, D'Orsi E, Benedetti TRB. Circunstâncias e consequências das quedas em idosos de Florianópolis. EpiFloripa Idoso 2009*. Rev Bras Epidemiol 2013; 16(2): 469-8.

10. Santos RKM, Maciel ÁCC, Britto HMJS, Lima JCC, Souza TO. Prevalência e fatores associados ao risco de quedas em idosos adscritos a uma Unidade Básica de Saúde do município de Natal, RN, Brasil. Ciência \& Saúde Coletiva 2015, 20(12):3753-3762.

11. Alves LV, Taguchi CK, Oliveira IL, Sousa MGC. Avaliação da tendência à quedas em idosos de Sergipe. Rev. CEFAC. 2014 Set-Out; 16(5):1389-1396.

12. Gai J, Gomes L, Nóbrega OT, Rodrigues MP. Fatores associados a quedas em mulheres idosas residentes na comunidade. Rev Assoc Med Bras 2010; 56(3): 327-32.

13. Coqueiro $R$, Santos $K$, Schettino $L$, Barbosa A, Pereira R, Fernandes M. "Chair Stand" Test as Predictor of Brazilian Elderly Men Fallers. Maced J Med Sci. 2014 Jun 15; 7(2):316318.

14. Gervásio FM, Barbosa AM, Brandão $M$, Gonçalves CS, Ribeiro DM, Junior JAST. et al. Marcha de Idosas e Risco de Quedas. Revista movimenta 2012. ISSN: 1984- 4298; 5(1): 40-54.

15. Santos GM, Souza ACS, Virtuoso JF, Tavares GMS, Mazo JZ. Valores preditivos para o rico de queda em idosos praticantes e não praticantes de atividade física por meio do uso da Escala de Equilíbrio de Berg. Rev Bras Fisioter. 2011; 15(2): 95-101.

16. Pimentel RM, Scheicher ME. Comparação do risco de queda em idosos sedentários e ativos por meio da escala de equilíbrio de Berg. Fisioterapia e Pesquisa 2009 jan./mar, São Paulo; 16(1): 6-10.

17. Mujdeci B, Aksoy S, Atas A. Evaluation of balance in fallers and non-fallers elderly. Brazilian Journal of Otorhinolaryngology 2012 setembro/outubro; 78(5): 104-109.

18. Falsarella GR, Gasparotto LPR, Coimbra AMV. Quedas: conceitos, frequências e aplicações à assistência ao idoso. Revisão da literatura. Rev. Bras. Geriatr. Gerontol., Rio de Janeiro, 2014; 17(4):897-910.

19. Gomes GAO, Cintra FA, Diogo MGD, Neri AL, Guariento ME, Sousa MLR. Comparação entre idosos que sofreram quedas segundo desempenho físico e número de ocorrências. Rev Bras Fisioter. 2009;13(5):430-7.
20. Ferraresi JR, Prata MG, Scheicher ME. Avaliação do equilíbrio e do nível de independência funcional de idosos da comunidade. Rev. Bras. Geriatr. Gerontol., Rio de Janeiro, 2015; 18(3):499-506.

21. Betran $O$, Júnior JES, Ribeiro $O R$, Corrente JE. Risk of falling among elderly persons living in the community: assessment by the Timed up and go test. Brazilian Journal of Otorhinolaryngology Janeiro/Fevereiro 2013; 79(1): 18-21.

22. Castro PMMA, Magalhães AM, Cruz ALC, Reis NSRD. Testes de equilíbrio e mobilidade funcional na predição e prevenção de riscos de quedas em idosos. Rev. Bras. Geriatr. Gerontol., Rio de Janeiro, 2015; 18(1):129-140.

\section{Endereço para Correspondência}

Faculdade Independente do Nordeste - FAINOR

Av. Luís Eduardo Magalhães, 1035 - Candeias, Vitória da Conquista - BA

CEP.: 45055-420

e-mail: kleyton_santos@hotmail.com

Recebido em 22/04/2017

Aprovado em 18/09/2017

Publicado em 30/11/2017 